\title{
Cytokines CCL2 and CXCL1 may be potential novel predictors of early bone loss
}

\author{
YAQIAN HU $^{1 *}$, LONG WANG $^{2 *}$, ZHUOJIE ZHAO $^{1}$, WEIGUANG LU ${ }^{1}$, JING FAN $^{1}$, \\ BO GAO $^{1}$, ZHUOJING LUO ${ }^{1}$, QIANG JIE ${ }^{3}$, XIAOJUAN SHI ${ }^{1}$ and LIU YANG $^{1}$ \\ ${ }^{1}$ Department of Orthopedic Surgery, Xijing Hospital, The Fourth Military Medical University, Xi'an, Shaanxi 710032; \\ ${ }^{2}$ Department of Orthopaedics, Chinese PLA General Hospital, Beijing 100853; ${ }^{3}$ Department of Orthopedic Surgery, \\ Honghui Hospital, College of Medicine, Xi'an Jiaotong University, Xi'an, Shaanxi 710049, P.R. China
}

Received November 21, 2019; Accepted June 18, 2020

DOI: $10.3892 / \mathrm{mmr} .2020 .11543$

\begin{abstract}
Osteoporosis is a common disorder characterized by decreased bone mineral density (BMD) and increased fracture risk. The current techniques detect real-time BMD precisely but do not provide adequate information to predict early bone loss. If bone loss could be diagnosed and predicted early, severe osteoporosis and unexpected fractures could be prevented, allowing for an improved quality of life for individuals. In the present study, an ovariectomized rat model of bone loss was established and the serum levels of 78 potential cytokines were determined using a protein array. The BMD of ovariectomized rats was dynamically measured by micro-CT and the early stage of bone loss was defined at the fourth week after surgery. The expression of several serum protein cytokines was indicated to be altered in the ovariectomized rats during an 8-week time-course of bone loss. Linear regression analysis revealed that the serum levels of C-C motif chemokine ligand 2 (CCL2, also known as monocyte chemoattractant protein 1) and C-X-C motif chemokine ligand 1 (CXCL1) were significantly associated with a reduction in BMD. The significance of these two factors in indicating bone mass reduction was further verified by analyzing serum samples from 24 patients with BMD using ELISA and performing a linear regression analysis. The serum levels of CCL2 and CXCL1 were inversely correlated with the bone mass. Therefore, the cytokines CCL2 and CXCL1 may be potential novel predictors of early bone loss and may be clinically relevant for the early diagnosis and prevention of osteoporosis.
\end{abstract}

Correspondence to: Dr Liu Yang or Dr Xiaojuan Shi, Department of Orthopedic Surgery, Xijing Hospital, The Fourth Military Medical University, 127 Changle West Road, Xi'an, Shaanxi 710032, P.R. China

E-mail: yangliu@fmmu.edu.cn

E-mail:shixj6166@163.com

${ }^{*}$ Contributed equally

Key words: bone loss, prediction, cytokines, C-C motif chemokine ligand 2, C-X-C motif chemokine ligand 1

\section{Introduction}

Osteoporosis is a common orthopedic disease featuring a decrease in bone mass and an increase in bone fragility. It is characterized by low bone mineral density (BMD), which is usually measured by traditional Dual energy X-ray methods or optimized methods (1). These classical methods are able to provide accurate BMD data; however, they cannot provide information that predicts early bone loss. On the other hand, if the early decline of bone mass can be identified, improved measures to prevent the occurrence and development of osteoporosis may be taken. Certain studies have focused their attention on exploring new measures to predict BMD by assessing bone turnover markers (2), C-C motif chemokine ligand (CCL)11/eotaxin-1 (3), calcium isotope (4), circular RNAs (5), metabolites (6), gene expression microarray analyses (7) and transforming growth factor- $\beta 3$ (8). If any of these factors were revealed to be able to indicate premature bone loss, they would hold great promise for the prevention of osteoporosis.

Inflammatory reactions are frequently accompanied by the occurrence of bone loss. The term osteoimmunology was adopted by Arron and Choi (9). Subsequently, it was demonstrated that interleukin (IL)-1 $\beta$ (10), IL-6 (11-15), IL-10 $(16,17)$ and IL-13 (18) are closely linked to osteoporosis. Other biomarkers were also indicated to be associated with BMD. For instance, early bone loss is frequently accompanied by changes in the body microenvironments, in particular, changes in serum cytokine levels, such as $\mathrm{CC}$ motif ligand 4 (CCL4)/macrophage inflammatory protein-1 $\beta$ (MIP-1 $\beta$ ) (19), sclerostin (20) and neuropeptide vasoactive intestinal peptide (21). Based on this serum screening method, the present study explored which of the various cytokines may be used as predictors of early bone loss.

In the present study, a rat model of ovariectomy-induced bone loss was successfully established to represent early osteoporosis in humans. The animals demonstrated progressive bone loss starting from four weeks after surgery. In the protein array screening, several cytokines in the rat serum were noted to be associated with the development of osteoporosis. Specifically, increased serum levels of CCL2 and C-X-C motif chemokine ligand 1 (CXCL1) were significantly 
associated with decreased BMD. Validation in clinical patient samples demonstrated that the cytokines CCL2 and CXCL1 were also significantly associated with reduced BMD in humans. Through linear regression analysis, linear regression equations between these two cytokines and changes in bone mass were obtained, from which the change in bone density may be predicted. Overall, the present study suggested that serum levels of CCL2 and CXCL1 could be used to predict the decline in BMD at an early stage in individuals in a rapid and non-invasive manner.

\section{Materials and methods}

Establishment of rat model of early bone loss. Female Sprague Dawley rats ( $\mathrm{n}=24$; age, 12 weeks; body weight, $240 \pm 16.9 \mathrm{~g}$ ) were purchased from the Experimental Animal Center of the Fourth Military Medical University (Xi'an, China). The rats were housed with free access to food and water $(12 \mathrm{~h}$ light/dark cycle, $20^{\circ} \mathrm{C}$ and $50-55 \%$ humidity). The rats were randomly divided into two groups: An ovariectomy group (OVX, $n=12$ ) and a sham group (Sham, $n=12)$. To establish the early bone loss model, all 12 rats in the OVX group underwent ovariectomy following anesthetization by intraperitoneal injection of pentobarbital at a dose of $40 \mathrm{mg} / \mathrm{kg}$. All 12 rats in the sham group underwent ovary ablation following the same anesthetization method. At 2, 4, 6 and 8 weeks after the surgical procedures, serum samples were collected from the animals in each group. All animal experimental procedures were approved by the Ethics in Experimental Animal Center of the Fourth Military Medical University (permission code IACUC-20190112).

Confirmation of early bone loss by $\mu C T$. At 2-week intervals over 2 months following the ovariectomy or sham operations (time-points of 2, 4, 6 and 8 weeks), each rat was anesthetized with pentobarbital at a dose of $40 \mathrm{mg} / \mathrm{kg}$ during $\mu \mathrm{CT}$ scanning. The American Society for Bone and Mineral Research recommendations for small-animal $\mu \mathrm{CT}$ (22) were followed during the process of $\mu \mathrm{CT}$ analysis. A pre-clinical Inveon $\mu \mathrm{CT}$ system (Siemens Healthineers) with a resolution of $8 \mathrm{~mm}$, tube current of $0.1 \mathrm{~mA}$ and tube voltage of $50 \mathrm{kV}$ was used to scan distal femurs. The three-dimensional quantitative analyses were performed using the $\mu \mathrm{CT}$ system (Inveon Research Workplace 2.2; Siemens Healthineers). Scanning regions were confined from the distal metaphysis and extended $2.0 \mathrm{~mm}$ proximally from the proximal tip of the primary spongiosa. The following three-dimensional indices in the defined region of interest were determined: $\mathrm{BMD}$, trabecular thickness (Tb.Th) and relative bone volume over the total volume (BV/TV, \%). The examiner performing the scan analyses was blinded to the experimental group of the subjects.

Serum cytokine array analyses. Blood samples $(1 \mathrm{ml})$ were obtained from the retro-orbital veins of each animal at 2, 4, 6 and 8 weeks following surgery. Rat serum samples collected every two weeks post-surgery were assessed for the presence of 78 cytokines (Table SI) using the Quantibody Rat Cytokine Array kit (RayBiotech Inc.) according to the manufacturer's protocol (Fig. S1). In brief, antibody array membranes were blocked with Tris-buffered saline supplemented with 5\% skimmed milk and $0.05 \%$ Tween- 20 for $1 \mathrm{~h}$, followed by the addition of rat serum to obtain final 10 -fold dilutions. The fluorescence brightness was detected and quantified on a fluorescent scanner (Axon GenePix; Molecular Devices, LLC) to determine the cytokine expression levels.

Human BMD detection and blood serum sample collection. A total of 24 individuals aged 15-75 years from Xijing Hospital (Xi'an, China) were recruited and provided informed signed consent, prior to being enrolled in the present study as human subjects. The experiment was approved by the Ethics Committee of Xijing Hospital of Fourth Military Medical University (Xi'an, China; permission code: XJYYLL-2014076). The demographic data of the patients are presented in Table SII. The patients had a normal hepatorenal function and were excluded if they had a history of cardiovascular disease, cancer or diabetes mellitus. Each human subject signed an informed consent document prior to study enrollment. None of the subjects had been diagnosed with any metabolic bone diseases nor had they been treated with any medications known to affect bone metabolism. In each subject, the BMD of the lumbar spine (L1-4) was measured using dual-energy X-ray absorptiometry scanners (QDR4500; Hologic, Inc.). Blood samples were collected in non-anticoagulated blood collection tubes at $4^{\circ} \mathrm{C}$ to obtain serum supernatants, which were then stored at $-80^{\circ} \mathrm{C}$ for further evaluation using ELISA.

Human serum ELISA and KEGG pathway maps. Human serum cytokine expression levels (CCL2 and CXCL1) were measured with ELISA assay kits (cat. nos. P13500 and P09341; RayBiotech, Inc.) according to the manufacturer's protocols. The total concentration of each cytokine was estimated using the Bradford protein assay method. In addition, Kyoto Encyclopedia of Genes and Genomes (KEGG) pathway analysis was used to identify possible pathways related to CCL2 and CXCL1 signaling in osteoporosis (23-25).

Statistical analysis. Statistical analyses were performed with SPSS software, version 22 (IBM Corp.). Quantitative data are expressed as the mean \pm standard deviation. Statistical tests were performed by two-way analysis of variance (ANOVA). ANOVA followed by the Bonferroni post-hoc test was performed for comparisons among multiple groups. A Pearson correlation was employed to determine the linear correlation between two variables. $\mathrm{P}<0.05$ was considered to indicate a statistically significant difference.

\section{Results}

Confirmation of early bone loss in an ovariectomized rat model. In order to track the occurrence of early bone loss, the BMDs and other bone parameters of rats were measured at two weeks following surgery. The rat distal femur structure was reconstructed based on CT scan images (Fig. 1A). At the fourth week after surgery, the BMD of the rats in the ovariectomized group began to decrease significantly. At the eighth week, the BMD decreased by $>15 \%$ (Fig. 1B). In the sham group, the BMD of rats did not change significantly at these time-points. Along with the decrease of BMD, quantitative analysis of 
A
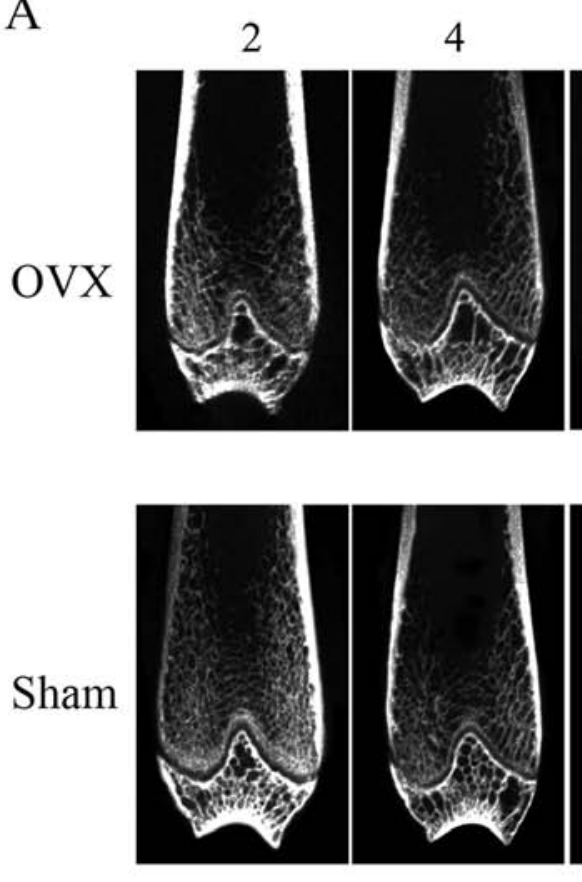

6
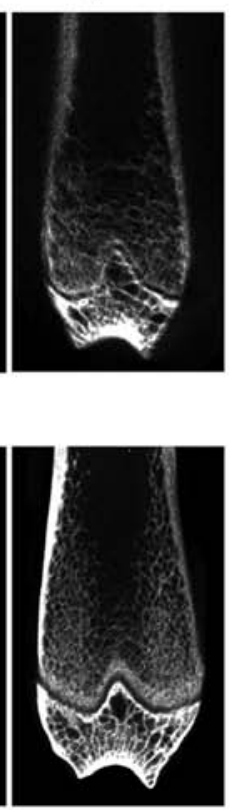

8 week (s)
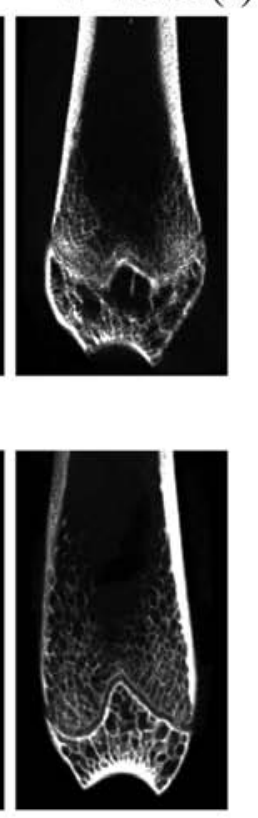
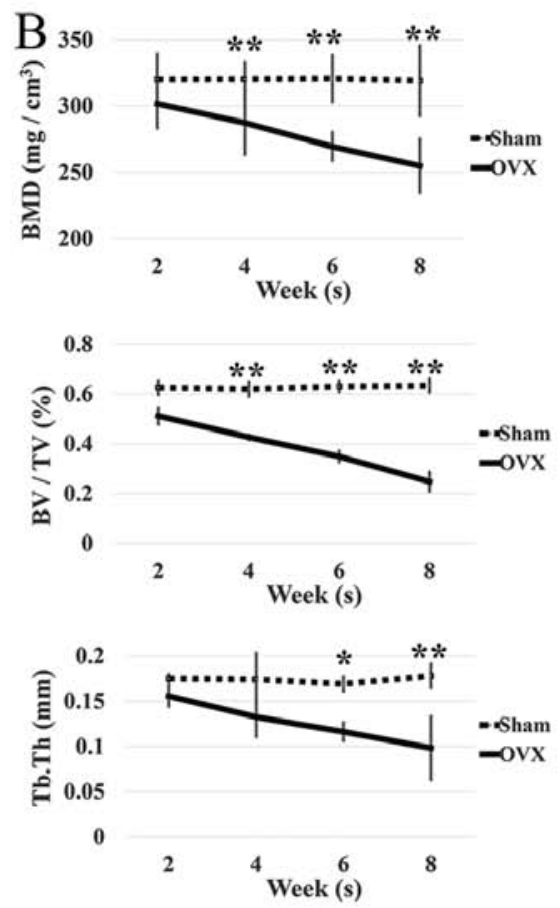

Figure 1. Establishment and confirmation of early osteoporosis in ovariectomized rat models. (A) $\mu \mathrm{CT}$ analysis within the metaphyseal distal femur region at 2, 4, 6 and 8 weeks following sham surgery or ovariectomy (original magnification, x40). Dynamic alterations of (B) BMD, BV/TV and Tb.Th in rats that received either sham surgery or ovariectomy. ${ }^{*} \mathrm{P}<0.05$ and ${ }^{* *} \mathrm{P}<0.01$, sham vs. OVX group ( $\mathrm{n}=5$ ). BMD, bone mineral density; BV/TV, relative bone volume over the total volume; Tb.Th, trabecular thickness; OVX, ovariectomy group.

further bone parameters in the ovariectomized group revealed a significant decrease in BV/TV at $4-8$ weeks (Fig. 1B) and Tb.Th at 6-8 weeks (Fig. 1B) post-surgery $(\mathrm{P}<0.05)$.

Screening for cytokines associated with early bone loss by protein array. A total of 78 serum cytokines were included in the protein array screening assay. By analysis of fluorescence intensities, it was revealed that in the OVX group, 20 serum cytokines increased or decreased steadily from 2 weeks post-surgery, while they remained unchanged in the Sham group. A total of 10 cytokines [proopiomelanocortin, $\beta$-catenin, CCL2, CXCL1, tumor necrosis factor super family member 6 , follistatin-like 1 , colony-stimulating factor 2 , CXCL6, matrix metalloproteinase (MMP)-8 and hypocretin neuropeptide precursor] were increased following ovariectomy (Fig. 2A), whereas another 10 cytokines (brain-derived neurotrophic factor, C-C motif chemokine receptor 4, C-X-C motif chemokine receptor 4, GDNF family receptor $\alpha 4$, IL-2, growth hormone 1, MMP-13, resistin-like $\beta$, Toll-like receptor 4 and IL-3) exhibited the opposite trend (Fig. 2B, Table SIII). The other 58 cytokines assessed showed insignificant changes between the OVX and the Sham group over the time-course (Fig. S2).

Linear regression analyses of cytokines and bone mass loss in the ovariectomized rat models. After protein array screening, 20 cytokines were noted to be elevated or decreased in the serum during the early stage of bone loss in the present model. However, whether they were also correlated with bone loss progression remained to be elucidated. To verify the association of these 20 cytokines with the progression of early bone loss, the correlation between the cytokine levels and BMD of ovariectomized rats was analyzed. Among the 20 cytokines, CCL2 and CXCL1 were inversely correlated with the BMD of the ovariectomized rats $(\mathrm{P}<0.05$, Fig. 3$)$. By contrast, the other 18 cytokines did not exhibit any significant correlations with BMD (P>0.05; Fig. S3, Table SIV).

Utility of CCL2 and CXCL1 in reflecting early bone mass reduction in human serum array. To further verify the clinical significance of the serum cytokines of CCL2 and CXCL1 as potential predictors of early bone loss, the present study detected the serum levels of human CCL2 and CXCL1 in clinical patients (mean age, 52.87 years old; female/male patients, 18/6; BMD for the subjects is provided in Table SII, 9 individuals had osteoporosis, 12 postmenopausal females were included) using commercially available ELISA kits. Linear regression analysis between CCL2 and CXCL1 levels and human BMD was then performed. The results demonstrated that these two candidate cytokines, CCL2 and CXCL1, were positively correlated with bone loss in humans ( $\mathrm{P}<0.05$; Fig. 4). The linear regression equation between CCL2 and BMD was $\mathrm{Y}\left(\mathrm{BMD}, \mathrm{mg} / \mathrm{cm}^{3}\right)=1,207.375-43.0247 * \mathrm{X}(\mathrm{CCL} 2, \mathrm{pg} / \mathrm{ml})$. The linear regression equation between $\mathrm{CXCL1}$ and BMD was $\mathrm{Y}$ $\left(\mathrm{BMD}, \mathrm{mg} / \mathrm{cm}^{3}\right)=2,025.413-1,085.2 * \mathrm{X}(\mathrm{CXCL} 1, \mathrm{pg} / \mathrm{ml})$. When the serum levels of CCL2 and CXCL1 increase to 6.2 and $1.0 \mathrm{pg} / \mathrm{ml}$, respectively, the BMD drops below $940 \mathrm{mg} / \mathrm{cm}^{3}$ (T-value <-1), which is considered to be indicative of early bone loss in the clinic. Applying these equations, CCL2 and CXCL1 serum levels may be used to deduce an individual's BMD value and predict premature bone loss in humans. In addition, KEGG pathway analysis revealed the involvement of typical osteoporosis-associated ILs in the same signaling pathways as CCL2 and CXCL1 (Figs. 5 and S4). Furthermore, 
A
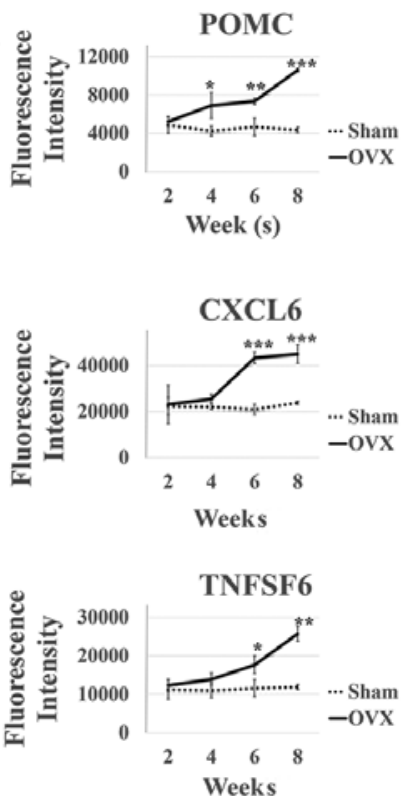

B

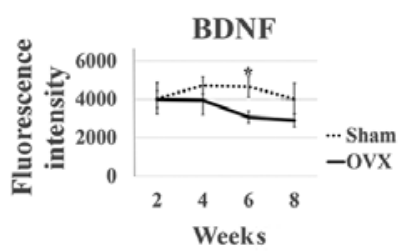

MMP-13

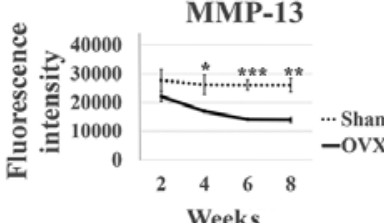

TLR4

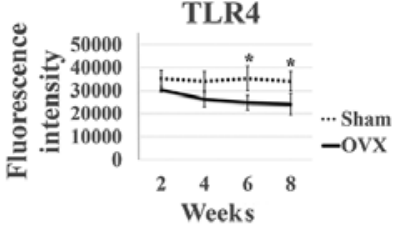

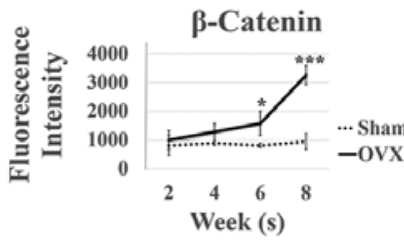

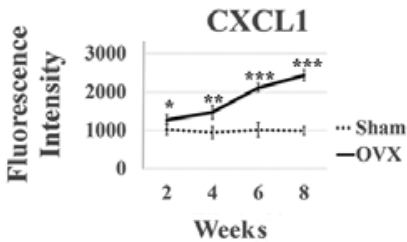

CCL2

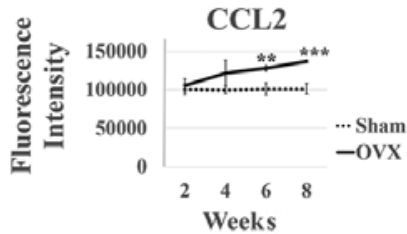

CXCR4

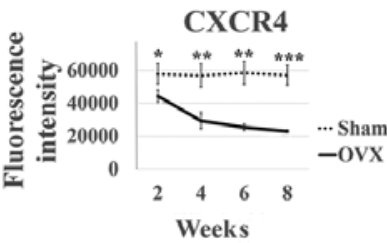

RETNLb

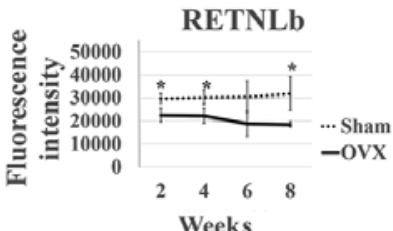

Weeks

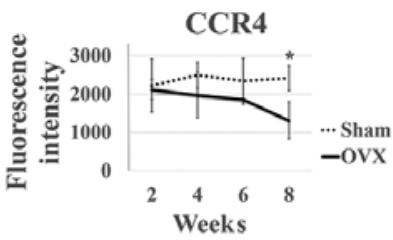

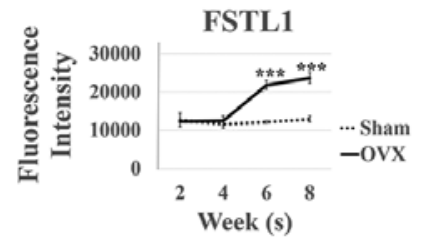
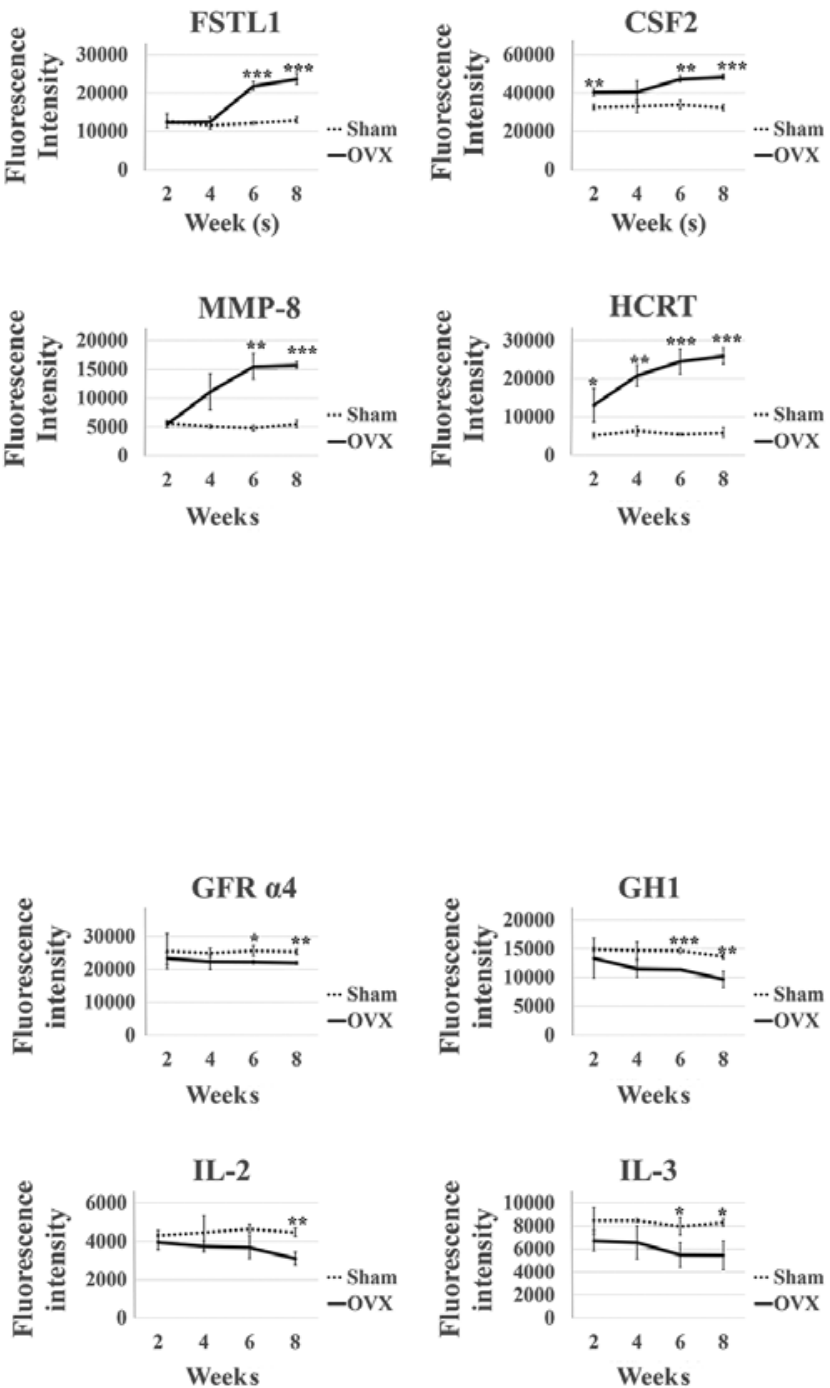

Figure 2. Screening results of bone loss-associated biomarkers using protein array. Dynamic alterations of serum cytokines in the sham or ovariectomized rats at 2, 4, 6 and 8 weeks following surgery. Each sample was repeatedly measured in three wells in the protein array. The fluorescence intensity was normalized by means of 8 control wells. (A) The cytokines that increased following ovariectomy. (B) The 10 cytokines that decreased following ovariectomy. ${ }^{*}<0.05$, ${ }^{* *} \mathrm{P}<0.01,{ }^{* * *} \mathrm{P}<0.001$ vs. sham group $(\mathrm{n}=3)$. OVX, ovariectomy group; CCL2, C-C motif chemokine ligand 2; CXCL1, C-X-C motif chemokine ligand 1; CXCR4, C-X-C motif chemokine receptor 4; MMP, matrix metalloproteinase; TLR, Toll-like receptor; IL, interleukin; BDNF, brain-derived neurotrophic factor; GFR, GDNF family receptor; CSF, colony-stimulatory factor; POMC, proopiomelanocortin; FSTL1, follistatin-like 1; HCRT, hypocretin neuropeptide precursor; TNSF; tumor necrosis factor superfamily member; GH, growth hormone.

according to the present results, these ILs were associated with decreased bone mass (Fig. S4).

\section{Discussion}

Osteoporosis is a common and severe degenerative disease. If osteoporosis were to be identified at its early stage, its development could be delayed and it would be possible to avoid substantial economic losses. In the present study, serum protein array screening in an ovariectomy-induced rat model of bone loss revealed that a total of 20 serum cytokines were aberrantly expressed in parallel with the development of early bone loss. Of the 20 potential markers, CCL2 and CXCL1 were further validated to be correlated with bone loss in the ovariectomy-induced osteoporosis rat model (resembled postmenopausal osteoporosis in humans). Postmenopausal osteoporosis is one of the most common skeletal diseases, for which practical methods for early diagnosis are lacking (3). Of note, in the present study, CCL2 and CXCL1 increased with the progression of postmenopausal osteoporosis, which may provide an effective and promising way to predict postmenopausal early bone loss. Further 
A CCL2

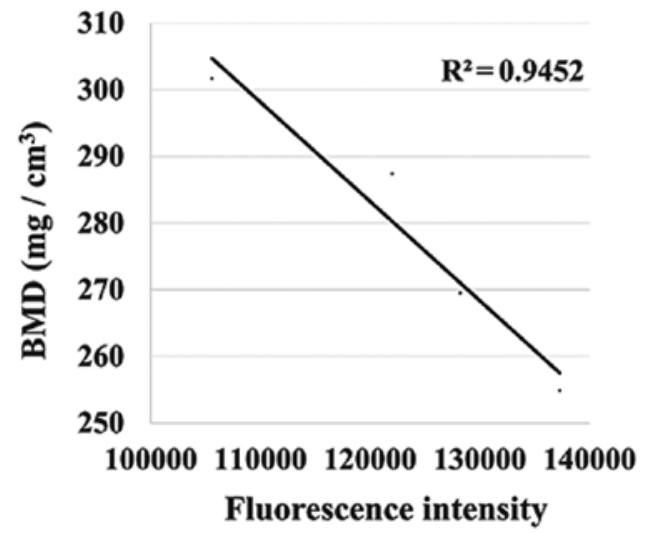

CXCL1

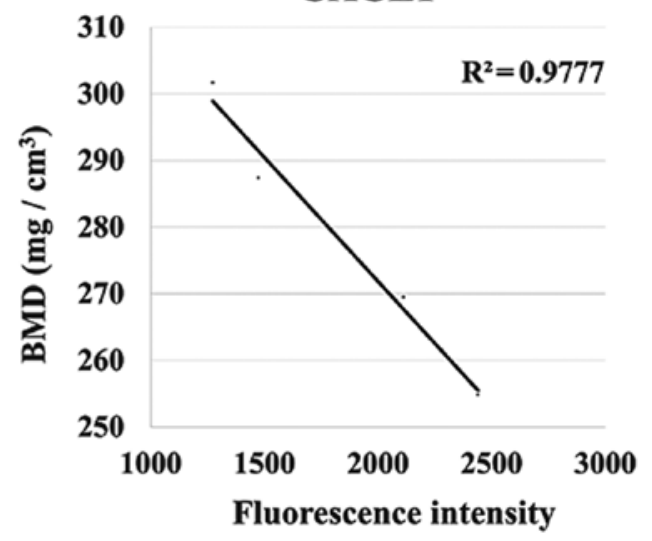

\begin{tabular}{|c|c|c|c|c|c|}
\hline \multicolumn{6}{|c|}{ Linear regression: $\mathbf{Y}(\mathbf{B M D})=A+B * X($ fluorescence intensity $)$} \\
\hline & & Value & Standard error & $T$ value & P-value \\
\hline \multirow{2}{*}{ CCL2 } & Intercept(A) & 461.9804 & 31.38874 & 14.71803 & \multirow{2}{*}{0.02777} \\
\hline & Slope(B) & -0.00149 & $2.54 \mathrm{E}-04$ & -5.87508 & \\
\hline \multirow{2}{*}{ CXCL1 } & Intercept(A) & 346.1606 & 7.47004 & 46.33985 & \multirow{2}{*}{0.01119} \\
\hline & Slope(B) & -0.03715 & 0.00396 & -9.37242 & \\
\hline
\end{tabular}

Figure 3. Correlation between serum CCL2 and CXCL1 levels and bone loss in rats. (A) CCL2 and CXCL1 were significantly negatively correlated with the BMD of ovariectomized rats $(\mathrm{P}<0.05)$. (B) Linear regression equation for the correlation of CCL2 and CXCL1 with BMD. CCL2, C-C motif chemokine ligand 2; CXCL1, C-X-C motif chemokine ligand 1; BMD, bone mineral density.

A

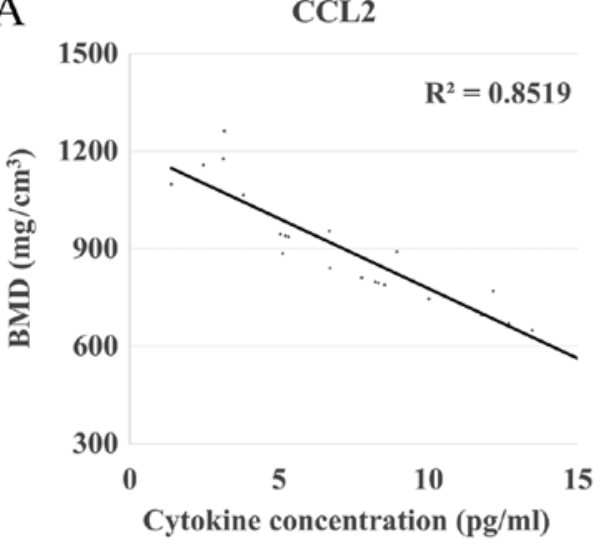

\section{CXCL1}

1500

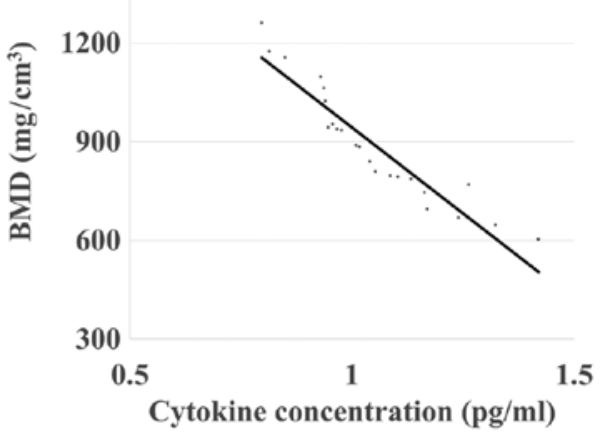

B

\begin{tabular}{|c|c|c|c|c|c|}
\hline \multicolumn{5}{|c|}{ Linear regression: $Y(B M D)=A+B * X$ (cytokine concentration) } \\
\hline & & Value & Standard error & T value & \multirow{2}{*}{ P-value } \\
\hline \multirow{3}{*}{ CCL2 } & Intercept(A) & 1207.375 & 31.02988 & 38.91009 & \multirow{2}{*}{0.0135981} \\
\cline { 2 - 5 } & Slope(B) & -43.0247 & 3.82457 & -11.2496 & \\
\hline \multirow{2}{*}{ CXCL1 } & Intercept(A) & 2025.413 & 79.50616 & 25.47492 & \multirow{2}{*}{0.03198} \\
\cline { 2 - 5 } & Slope(B) & -1085.2 & 75.63863 & -14.3472 & \\
\hline
\end{tabular}

Figure 4. Validation of CCL2 and CXCL1 in human serum samples. (A) CCL2 and CXCL1 were significantly negatively correlated with human BMD (P<0.05). (B) Linear regression equation for the correlation of CCL2 and CXCL1 with BMD. CCL2, C-C motif chemokine ligand 2; CXCL1, C-X-C motif chemokine ligand 1; BMD, bone mineral density. 


\begin{tabular}{|c|l|l|c|}
\hline A & \multicolumn{1}{|c|}{ Pathway } & ID & Web Links \\
\hline 1 & Cytokine-cytokine receptor interaction & $\mathbf{0 4 0 6 0}$ & https://www.kegg.jp/pathway/hsa04060 \\
\hline 2 & NOD-like receptor signaling pathway & $\mathbf{0 4 6 2 1}$ & https://www.kegg.jp/pathway/hsa04621 \\
\hline 3 & Influenza A & $\mathbf{0 5 1 6 4}$ & https://www.kegg.jp/pathway/hsa05164 \\
\hline 4 & Rheumatoid arthritis & $\mathbf{0 5 3 2 3}$ & http://www.kegg.jp/pathway/hsa05323 \\
\hline
\end{tabular}

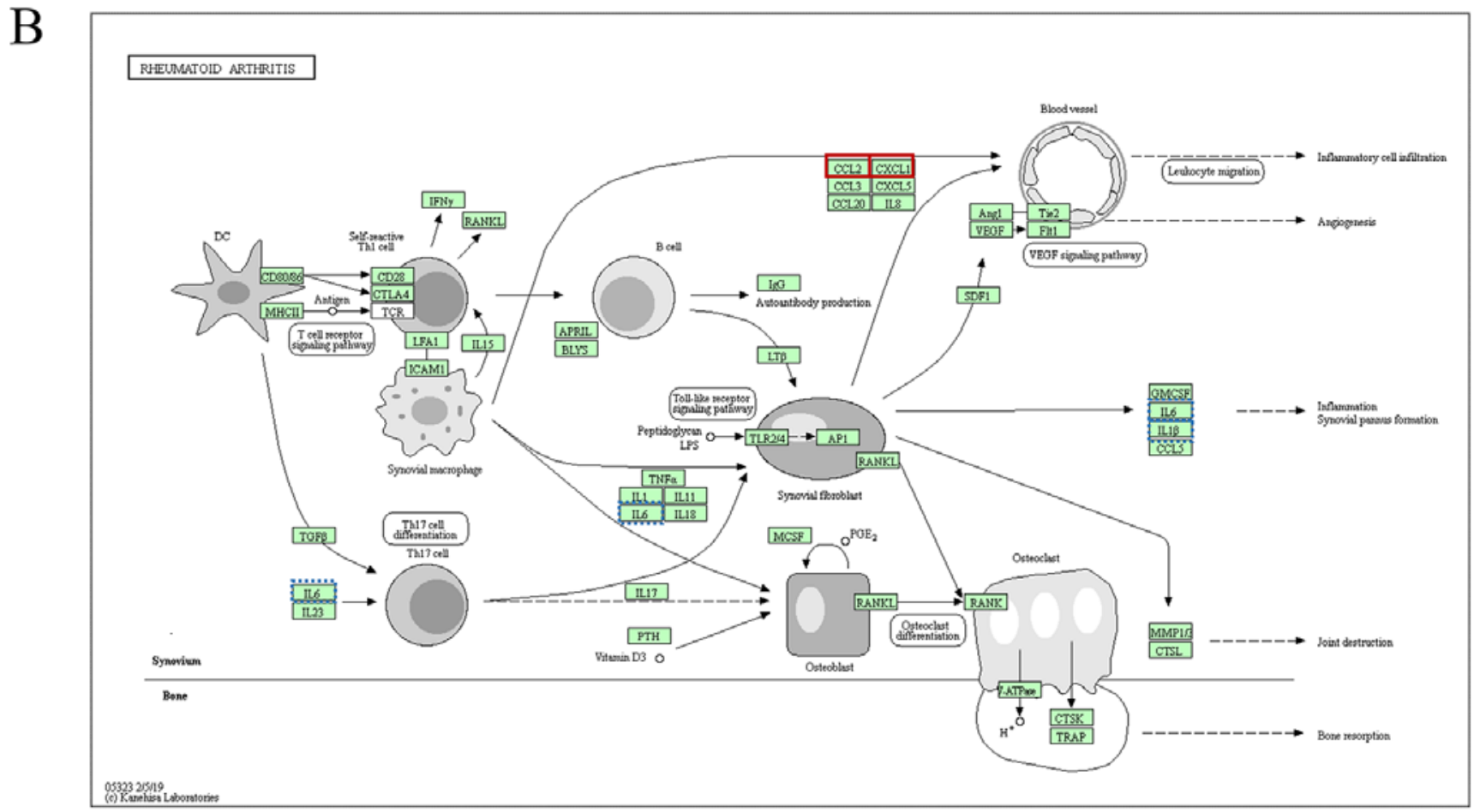

Figure 5. CCL2, CXCL1 and certain ILs are jointly involved in certain pathways. (A) Pathway information. (B) Rheumatoid arthritis pathway. Red solid box: CCL2 and CXCL1 (linear correlation with BMD significantly). Blue dotted boxes denote IL-1 $\beta$ and IL-6 (insignificant changes over the time-course). CCL2, C-C motif chemokine ligand 2; CXCL1, C-X-C motif chemokine ligand 1; IL, interleukin.

statistical analysis of results obtained with human subjects suggested that this novel prediction method may be used for early diagnosis of osteoporosis, which may assist in the implementation of interventions for osteoporosis in a timely manner. The human subjects of the present study were not only patients with postmenopausal osteoporosis, but the cohort reflected age-associated osteoporosis, indicating that these two predictive markers may be universally applicable for age-associated osteoporosis.

CCL2 and CXCL1 belong to the superfamily of chemokines. CCL2 (also known as monocyte chemoattractant protein 1) is a secreted protein involved in immunoregulatory and inflammatory processes. CCL2 has roles in systemic inflammation (26), enhancing the efficacy of immunotherapy (27) and also promoting the migration of tumor cells and macrophage-like cells (28). CXCL1 is a member of the CXC subfamily of chemokines and has a role in inflammation as a chemoattractant for neutrophils. CXCL1 may promote the proliferation of neural stem cells (29), restore neutrophil migration (30) and inhibit paclitaxel-induced peripheral neuropathy in mice (31). In the inflammatory response, these two factors may be detected simultaneously, suggesting that they may also function together. Respective induction of CXCL1 and CCL2 in spinal cord astrocytes and neurons may contribute to neuropathic pain (32). Neuroinflammation in chronic pain conditions involves CCL2 and CXCL1 and recent studies suggested that bone marrow stem cells (BMSCs) produced potent analgesic effects in animal models of inflammatory pain, neuropathic pain and cancer pain (33). Microglia-derived IL-1 $\beta$ promoted CCL2 and CXCL1 expression by Müller cells in focal retinal degeneration (34).

Current bone-associated studies have indicated a definite relationship between bone marrow/BMSCs and CCL2. Enhanced levels of CCL2 were discovered in the bone marrow of septic mice (35). B-cell acute lymphoblastic leukemia cell-derived CCL2 was reported to increase periostin levels in BMSCs (36). The present study also confirmed the role of CCL2 in bone, as the serum levels of CCL2 reflected changes in bone loss. Furthermore, a linear correlation between CXCL1 and the decline in BMD was revealed in the present 
study, while there are currently only a few reports on the correlation between CXCL1 and bone mass (37). The specificity of CCL2 and CXCL2 for osteoporosis may be limited, as they are general chemokines that may not be specific for osteoporosis. This research should be further assessed in the future. Through retrieval of pathway information from KEGG, it was revealed that CCL2, CXCL1 and numerous ILs are involved in certain pathways together. Previous studies indicated that IL-1 $\beta$ and IL- 6 are significantly associated with osteoporosis (38). In the serum cytokine array of the present study, they all displayed a significant decreasing trend from two to six weeks after surgery, followed by an increasing trend through to the eighth week. From these results, it may be expected that during the process of bone loss, the body's inflammatory response also changes from an initial decrease to a delayed rise, and finally, there would be significant inflammation during osteoporosis $(39,40)$. However, this phenomenon requires to be evidenced in future studies.

Estrogen deficiency, systemic inflammation and these two cytokines (CCL2 and CXCL1) are closely linked and these two cytokines have been reported to respond to systemic inflammation $(26,41)$. Although estrogen deficiency may induce higher CCL2 expression levels (42), the present study revealed that the trends of these two cytokines were still present in age-associated osteoporosis. Accordingly, it may be more likely that CCL2 and CXCL1 are indicators of systemic inflammation rather than estrogen deficiency. Furthermore, studies demonstrated that CCL2 and CXCL1 were involved in physiological bone remodeling, CCL2 was primarily expressed by bone-forming osteoblasts (43) and these two cytokines mediated osteoclastogenesis and accelerated osteoclast maturation $(37,44-46)$. These results may explain why the progress of bone loss was accompanied by increasing serum levels of CCL2 and CXCL1 in the present study.

In previous studies exploring BMD prediction methods, the genetic risk score was assessed (47) and a genome-wide association study was performed (48), which were costly and DNA sequencing was tedious. Certain studies have also made rigorous attempts to identify associations between serum markers and bone loss. In a 10-year follow-up study based on a large cohort of human subjects, investigators failed to identify a significant association between serum testosterone and bone mass loss (49). The present trial was based on a small cross-sectional cohort of 24 subjects and there were limitations of validation and long-term follow-up. Verification of the present method in a large-sample population and application of clinical testing in the future may improve the quality of life of patients while lowering costs and save time.

In previous studies on osteoporosis, real-time bone density was measured, but there is currently a lack of tools to monitor its changes, making it difficult to provide accurate predictions on osteoporosis development. If it were possible to use serum cytokines for predicting bone mass reduction, this may provide advantages of convenience, accuracy and efficiency, while avoiding the risk of radiation damage from other methods. The present results suggested that serum levels of CCL2 and CXCL1 may be used as a novel tool for early diagnosis and early intervention of osteoporosis.

\section{Acknowledgements}

Not applicable.

\section{Funding}

This work was funded by the National Natural Science Foundation of China (grant nos. 81772377 and 81730065) and the Natural Science Foundation of Shaanxi Province (grant no. 2017ZDJC-12).

\section{Availability of data and materials}

The datasets used and/or analyzed during the current study are available from the corresponding author on reasonable request.

\section{Authors' contributions}

YH, LY and XS designed the study; YH and LW performed experiments; ZZ and ZL acquired data; WL, QJ, BG and JF analyzed and interpreted data; YH and LY wrote the manuscript. All authors read and approved the final manuscript.

\section{Ethics approval and consent to participate}

All applicable international, national, and/or institutional guidelines for the care and use of animals were followed. All animal experimental procedures were approved by the Ethics in Experimental Animal Center of the Fourth Military Medical University (permission code IACUC-20190112). All procedures involving human participants were in accordance with the ethical standards of the institutional and/or national research committee and with the 1964 Helsinki declaration and its later amendments or comparable ethical standards. The experiment involving patients was approved by the Ethics Committee of Xijing Hospital of Fourth Military Medical University (Xi'an, China; permission code: XJYYLL-2014076). Informed consent was obtained from all individual participants included in the study.

\section{Patient consent for publication}

Not applicable.

\section{Competing interests}

The authors declare that they have no competing interests.

\section{References}

1. López Picazo M, Humbert L, Di Gregorio S, González Ballester MA and Del Río Barquero LM: Discrimination of osteoporosis-related vertebral fractures by DXA-derived 3D measurements: A retrospective case-control study. Osteoporosis Int 30: 1099-1110, 2019.

2. Gutierrez-Buey G, Restituto P, Botella S, Monreal I, Colina I, Rodríguez-Fraile M, Calleja A and Varo N: Trabecular bone score and bone remodelling markers identify perimenopausal women at high risk of bone loss. Clin Endocrinol (Oxf) 91: 391-399, 2019.

3. Wang W, Huang CY, Wang ZP, Xu SS, Qian TY, Chen YD and Wu WG: Serum C-C motif ligand 11/eotaxin-1 may serve as a candidate biomarker for postmenopausal osteoporosis. J Med Biochem 38: 353-360, 2019. 
4. Eisenhauer A, Müller M, Heuser A, Kolevica A, Glüer CC Both M, Laue C, Hehn UV, Kloth S, Shroff R and Schrezenmeir J: Calcium isotope ratios in blood and urine: A new biomarker for the diagnosis of osteoporosis. Bone Rep 10: 100200, 2019.

5. Huang Y, Xie J and Li E: Comprehensive circular RNA profiling reveals circ 0002060 as a potential diagnostic biomarkers for osteoporosis. J Cell Biochem 120: 15688-15694, 2019.

6. Wang J, Yan D, Zhao A, Hou X, Zheng X, Chen P, Bao Y, Jia W, Hu C, Zhang ZL and Jia W: Discovery of potential biomarkers for osteoporosis using LC-MS/MS metabolomic methods. Osteoporosis Int 30: 1491-1499, 2019.

7. Yang C, Ren J, Li B, Jin C, Ma C, Cheng C, Sun Y and Shi X: Identification of gene biomarkers in patients with postmenopausal osteoporosis. Mol Med Rep 19: 1065-1073, 2019.

8. Haghighizadeh E, Shahrezaee M, Sharifzadeh S and Momeni M: Transforming growth factor- $\beta 3$ relation with osteoporosis and osteoporotic fractures. J Res Med Sci 24: 46, 2019.

9. Arron JR and Choi Y: Bone versus immune system. Nature 408: 535-536, 2000.

10. Chao TH, Yu HN, Huang CC, Liu WS, Tsai YW and Wu WT: Association of interleukin-1 beta $(-511 \mathrm{C} / \mathrm{T})$ polymorphisms with osteoporosis in postmenopausal women. Ann Saudi Med 30: 437-441, 2010

11. Wakabayashi H, Kato S, Nagao N, Miyamura G, Naito Y and Sudo A: Interleukin-6 inhibitor suppresses hyperalgesia without improvement in osteoporosis in a mouse pain model of osteoporosis. Calcified Tissue Int 104: 658-666, 2019.

12. Harmer D, Falank C and Reagan MR: Interleukin-6 interweaves the bone marrow microenvironment, bone loss, and multiple myeloma. Front Endocrinol (Lausanne) 9: 788, 2018.

13. Kim HJ, Kim HJ, Choi Y, Bae MK, Hwang DS, Shin SH and Lee JY: Zoledronate enhances osteocyte-mediated osteoclast differentiation by IL-6/RANKL axis. Int J Mol Sci 20: 1467, 2019.

14. Edwards CJ and Williams E: The role of interleukin-6 in rheumatoid arthritis-associated osteoporosis. Osteoporosis Int 21 1287-1293, 2010

15. Blumenfeld O, Williams FMK, Valdes A, Hart DJ, Malkin I, Spector TD and Livshits G: Association of interleukin-6 gene polymorphisms with hand osteoarthritis and hand osteoporosis. Cytokine 69: 94-101, 2014

16. Tousen Y, Matsumoto Y, Nagahata Y, Kobayashi I, Inoue M and Ishimi Y: Resistant starch attenuates bone loss in ovariectomised mice by regulating the intestinal microbiota and bone-marrow inflammation. Nutrients 11: 297, 2019.

17. Kotrych D, Dziedziejko V, Safranow K, Sroczynski T, Staniszewska M, Juzyszyn Z and Pawlik A: TNF- $\alpha$ and IL10 gene polymorphisms in women with postmenopausal osteoporosis. Eur J Obstet Gynecol Reprod Biol 199: 92-95, 2016.

18. Ding Q, Zhou H, Yun B, Zhou L, Zhang N, Yin G and Fan J: Interleukin-13 inhibits expression of cyp27b1 in peripheral CD14+ cells that is correlated with vertebral bone mineral density of patients with ulcerative colitis. J Cell Biochem 118: 376-381, 2017.

19. Yang XW, Wang F, Qin RZ, Zhou QL and Huang HX: Elevated serum CCL4/MIP-1 $\beta$ levels in postmenopausal osteoporosis patients are linked with disease severity. Biomark Med 13: 17-25, 2019.

20. Zheng J, Maerz W, Gergei I, Kleber M, Drechsler C, Wanner C, Brandenburg V, Reppe S, Gautvik KM, Medina-Gomez C, et al: Mendelian randomization analysis reveals a causal influence of circulating sclerostin levels on bone mineral density and fractures J Bone Miner Res 34: 1824-1836, 2019.

21. Wang W, Wang ZP, Huang CY, Chen YD, Yao WF and Shi BM: The neuropeptide vasoactive intestinal peptide levels in serum are inversely related to disease severity of postmenopausal osteoporosis: A cross-sectional study. Genet Test Mol Bioma 23: 480-486, 2019.

22. Vollherbst DF, Otto R, Do T, Kauczor HU, Bendszus M, Sommer CM and Möhlenbruch MA: Imaging artifacts of Onyx and PHIL on conventional CT, cone-beam CT and MRI in an animal model. Interv Neuroradiol 24: 693-701, 2018.

23. Kanehisa M and Goto S: KEGG: Kyoto encyclopedia of genes and genomes. Nucleic Acids Res 28: 27-30, 2000.

24. Kanehisa M, Sato Y, Furumichi M, Morishima K and Tanabe M: New approach for understanding genome variations in KEGG. Nucleic Acids Res 47 (D1): D590-D595, 2019.

25. Kanehisa M: Toward understanding the origin and evolution of cellular organisms. Protein Sci 28: 1947-1951, 2019.
26. Krause K, Sabat R, Witte Händel E, Schulze A, Puhl V, Maurer M and Wolk K: Association of CCL2 with systemic inflammation in Schnitzler syndrome. Br J Dermatol 180: 859-868, 2019.

27. Wang Y, Zhang X, Yang L, Xue J and Hu G: Blockade of CCL2 enhances immunotherapeutic effect of anti-PD1 in lung cancer. J Bone Oncol 11: 27-32, 2018.

28. Higashino N, Koma YI, Hosono M, Takase N, Okamoto M, Kodaira H, Nishio M, Shigeoka M, Kakeji Y and Yokozaki H: Fibroblast activation protein-positive fibroblasts promote tumor progression through secretion of CCL2 and interleukin-6 in esophageal squamous cell carcinoma. Lab Invest 99: 777-792, 2019.

29. Shang Y, Tian L, Chen T, Liu X, Zhang J, Liu D, Wei J, Fang W, Chen Y and Shang D: CXCL1 promotes the proliferation of neural stem cells by stimulating the generation of reactive oxygen species in APP/PS1 mice. Biochem Biophys Res Commun 515: 201-206, 2019.

30. Salinas-Muñoz L, Campos-Fernández R, Olivera-Valle I, Mercader E, Fernandez-Pacheco C, Lasarte S, Pérez-Martín L, Navarro-González MT and Sánchez-Mateos: Estradiol impairs epithelial CXCL1 gradient in the cervix to delay neutrophil transepithelial migration during insemination. J Reprod Immunol 132: 9-15, 2019.

31. Manjavachi MN, Passos GF, Trevisan G, Araújo SB, Pontes JP, Fernandes ES, Costa R and Calixto JB: Spinal blockage of CXCL1 and its receptor CXCR2 inhibits paclitaxel-induced peripheral neuropathy in mice. Neuropharmacology 151: 136-143, 2019.

32. Zhang ZJ, Cao DL, Zhang X, Ji RR and Gao YJ: Chemokine contribution to neuropathic pain: Respective induction of CXCL1 and CXCR2 in spinal cord astrocytes and neurons. Pain 154: 2185-2197, 2013

33. Huh Y, Ji RR and Chen G: Neuroinflammation, bone marrow stem cells, and chronic pain. Front Immunol 8: 1014, 2017.

34. Natoli R, Fernando N, Madigan M, Chu-Tan JA, Valter K, Provis J and Rutar M: Microglia-derived IL-1 $\beta$ promotes chemokine expression by Müller cells and RPE in focal retinal degeneration. Mol Neurodegener 12: 31, 2017.

35. Smirnov A, Pohlmann S, Nehring M, Ali S, Mann-Nüttel R, Scheu S, Antoni AC, Hansen W, Büettner M, Gardiasch MJ, et al: Sphingosine 1-phosphate- and C-C chemokine receptor 2-dependent activation of CD4+ plasmacytoid dendritic cells in the bone marrow contributes to signs of sepsis-induced immunosuppression. Front Immunol 8: 1622, 2017.

36. Ma Z, Zhao X, Deng M, Huang Z, Wang J, Wu Y, Cui D, Liu Y, Liu R and Ouyang G: Bone marrow mesenchymal stromal cell-derived periostin promotes $\mathrm{B}$-all progression by modulating CCL2 in leukemia cells. Cell Rep 26: 1533-1543.e4, 2019.

37. Hardaway AL, Herroon MK, Rajagurubandara E and Podgorski I Marrow adipocyte-derived CXCL1 and CXCL2 contribute to osteolysis in metastatic prostate cancer. Clin Exp Metastasis 32: 353-368, 2015.

38. Di Munno O and Ferro F: The effect of biologic agents on bone homeostasis in chronic inflammatory rheumatic diseases. Clin Exp Rheumatol 37: 502-507, 2019.

39. Lacativa PG and Farias ML: Osteoporosis and inflammation. Arq Bras Endocrinol Metabol 54: 123-132, 2010.

40. Mundy GR: Osteoporosis and inflammation. Nutr Rev 65: S147-S151, 2007.

41. Babu H, Ambikan AT, Gabriel EE, Svensson Akusjärvi S, Palaniappan AN, Sundaraj V, Mupanni NR, Sperk M, Cheedarla N, Sridhar R, et al: Systemic inflammation and the increased risk of inflamm-aging and age-associated diseases in people living with HIV on long term suppressive antiretroviral therapy. Front Immunol 10: 1965, 2019.

42. Ke JY, Kliewer KL, Hamad EM, Cole RM, Powell KA, Andridge RR, Straka SR, Yee LD and Belury MA: The flavonoid, naringenin, decreases adipose tissue mass and attenuates ovariectomy-associated metabolic disturbances in mice. Nutr Metab (Lond) 12: 1, 2015

43. Siddiqui JA and Partridge NC: CCL2/Monocyte chemoattractant protein 1 and parathyroid hormone action on bone. Front Endocrinol (Lausanne) 8: 49, 2017.

44. Valerio MS, Herbert BA, Basilakos DS, Browne $\mathrm{C}, \mathrm{Yu} \mathrm{H}$ and Kirkwood KL: Critical role of MKP-1 in lipopolysaccharide-induced osteoclast formation through CXCL1 and CXCL2. Cytokine 71: 71-80, 2015

45. Khan UA, Hashimi SM, Bakr MM, Forwood MR and Morrison NA: CCL2 and CCR2 are essential for the formation of osteoclasts and foreign body giant cells. J Cell Biochem 117: 382-389, 2016. 
46. Chen W, Foo SS, Taylor A, Lulla A, Merits A, Hueston L, Forwood MR, Walsh NC, Sims NA, Herrero LJ and Mahalingam S: Bindarit, an inhibitor of monocyte chemotactic protein synthesis, protects against bone loss induced by chikungunya virus infection. J Virol 89: 581-593, 2015.

47. Ho-Le TP, Center JR, Eisman JA, Nguyen HT and Nguyen TV: Prediction of bone mineral density and fragility fracture by genetic profiling. J Bone Miner Res 32: 285-293, 2017.

48. Ho-Le TP, Pham HM, Center JR, Eisman JA, Nguyen HT and Nguyen TV: Prediction of changes in bone mineral density in the elderly: Contribution of 'osteogenomic profile'. Arch Osteoporos 13: 68, 2018.
49. Orwoll ES,Lapidus J, Wang PY, Vandenput L, Hoffman A, Fink HA, Laughlin GA, Nethander M, Ljunggren Ö, Kindmark A, et al: The limited clinical utility of testosterone, estradiol, and sex hormone binding globulin measurements in the prediction of fracture risk and bone loss in older men. J Bone Miner Res 32: 633-640, 2017.

(i) (2) This work is licensed under a Creative Commons Attribution-NonCommercial-NoDerivatives 4.0 International (CC BY-NC-ND 4.0) License. 